\title{
Transatlantica
}

Revue d'études américaines. American Studies Journal

$2 \mid 2011$

Sport et société / Animals and the American

Imagination

\section{Art contemporain ou kitsch ? Les productions de Gregory Crewdson}

\section{Bernard Genton}

\section{(2) OpenEdition}

\section{Journals}

Édition électronique

URL : https://journals.openedition.org/transatlantica/5610

DOI : 10.4000/transatlantica.5610

ISSN : 1765-2766

Éditeur

Association française d'Etudes Américaines (AFEA)

Référence électronique

Bernard Genton, «Art contemporain ou kitsch ? Les productions de Gregory Crewdson »,

Transatlantica [En ligne], 2 | 2011, mis en ligne le 14 mai 2012, consulté le 01 février 2023. URL : http:// journals.openedition.org/transatlantica/5610; DOI : https://doi.org/10.4000/transatlantica.5610

Ce document a été généré automatiquement le 1 février 2023.

\section{(c)}

Creative Commons - Attribution - Pas d'Utilisation Commerciale - Pas de Modification 4.0 International - CC BY-NC-ND 4.0

https://creativecommons.org/licenses/by-nc-nd/4.0/ 


\section{Art contemporain ou kitsch ? Les productions de Gregory Crewdson}

\section{Bernard Genton}

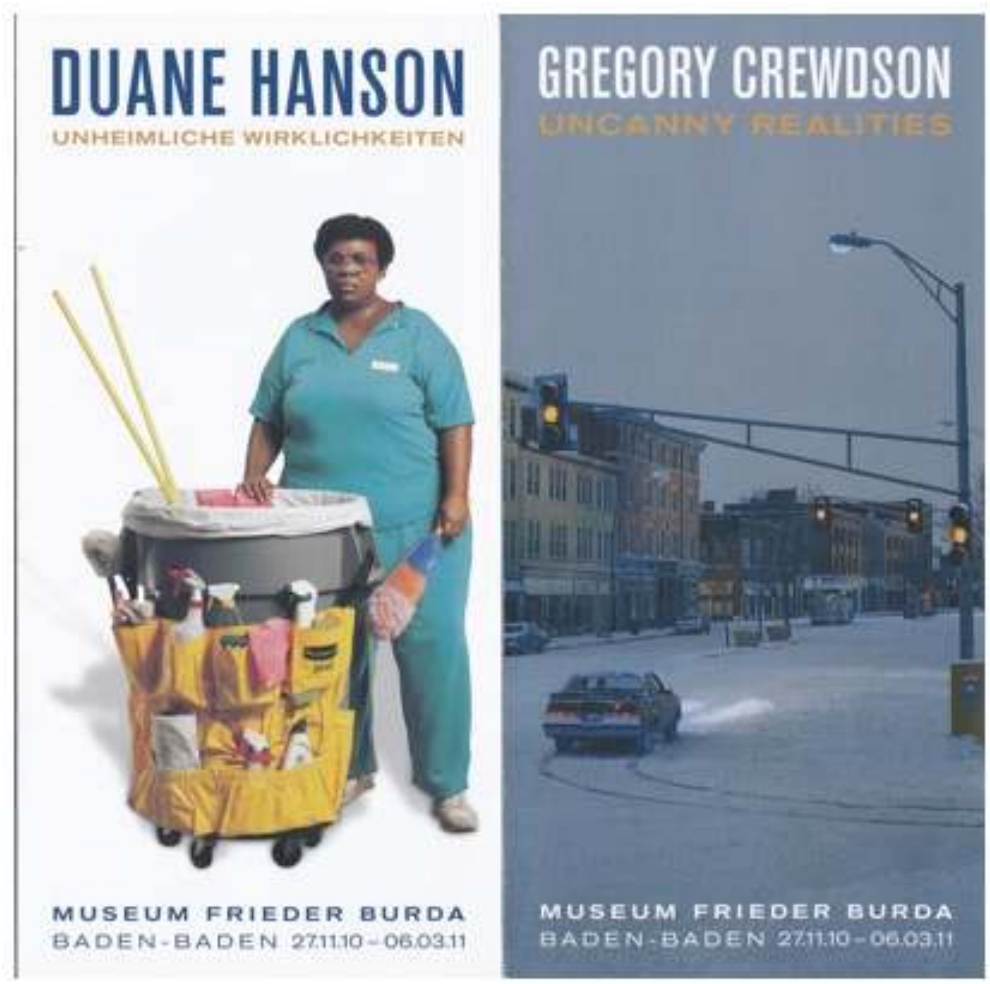

1 Le point de départ de la présente réflexion est l'exposition présentée au musée Burda de Baden-Baden au cours de l'hiver 2010-2011. Sous le titre trilingue L'inquiétante étrangeté de réel/Uncanny Realities/Unheimliche Wirklichkeiten on trouvait là, dans cette belle galerie imaginée par Richard Meier, 25 œuvres de Duane Hanson (1925-1996) dont beaucoup sont connues, face à 20 tirages de Gregory Crewdson (né en 1962), sans que le rapport entre les deux artistes soit évident. Des sculptures à échelle humaine, des tirages monumentaux $(144,8 \times 223,5 \mathrm{~cm})$, avec pour ces derniers, en sus du 
commentaire audio, une profusion d'explications, de graphiques, de photographies de plateau, de dessins originaux, de schémas des dispositifs d'éclairage, ainsi que plusieurs ouvrages déjà édités (il n'y avait pas de catalogue), des posters de tailles et de prix divers, encadrés ou non, et un choix limité de cartes postales.

Résumons l'essentiel : né à Brooklyn en 1962, Crewdson grandit dans une famille de la classe moyenne supérieure (son père est psychanalyste). Après une licence d'études artistiques à l'Université de l'État de New York à Purchase, il obtient un master à Yale. Crewdson enseigne ensuite dans plusieurs universités de la côte Est avant de rejoindre le corps professoral de Yale en 1993. Son travail photographique a été exposé une cinquantaine de fois depuis 1991, et ses œuvres ont fait partie de plus d'une centaine d'expositions collectives aux États-Unis, en Europe, mais aussi en Australie et au Japon ${ }^{1}$. Plusieurs grands musées américains ont acquis des tirages, tout comme un nombre appréciable d'institutions extérieures aux États-Unis. Crewdson, désormais représenté par la prestigieuse galerie Gagosian à New York, figure parmi les 400 photographes qui comptent ou ont compté retenus par le dictionnaire encyclopédique récemment publié sous la direction de Hans-Michael Koetzle² .

3 L'œuvre de Gregory Crewdson se développe en plusieurs phases successives, soigneusement ordonnées dans l'ouvrage rétrospectif qui paraît en $2005^{3}$. On y distingue les premiers travaux de la période 1986-1988 (Early Work), une série d'images végétales ou animales réalisées entre 1992 et 1997 (Natural Wonder) puis, en 1996-1997, un travail en noir et blanc intitulé Hover, qui présente des vues plongeantes, produites du haut d'une grue, d'un paysage suburbain de l'ouest du Massachusetts. C'est avec les cycles Twilight (1998-2002), Dream House (2002) et Beneath the Roses (2005) ${ }^{4}$ - ce dernier fournissant les 20 images présentées à Baden-Baden - que les "productions" de Crewdson atteignent pour ainsi dire leur plénitude formelle (grands formats en couleur, manipulations et retouches numériques, lumières) et thématique (provinces abandonnées, ambiances, intérieurs et extérieurs, traitement des personnages) ${ }^{5}$.

Admettons-le : les photographies de Gregory Crewdson sont d'un abord séduisant. Le spectateur reconnaît en elles un ensemble de signes déjà vus ailleurs et qui manifestent une "Amérique » familière à la fois attirante et vaguement inquiétante. Si l'on s'en tient aux images de la série Beneath the Roses, la plus aboutie, on est immédiatement conforté par la vue de ces carrefours déserts de petites villes à l'aube, ces paysages de supérettes, ces intérieurs ordinaires, ces chambres à coucher avec vue sur la salle de bain et personnages nus, en sous-vêtements ou en pyjama, ces territoires semi-urbains où des promeneurs en tenue d'été (dont la taille a été réduite par des manipulations numériques) longent des voies ferrées désaffectées. Dans l'une des deux déclinaisons de ce thème, la tranquillité est perturbée par l'incendie d'une vieille bâtisse en bois, mais les flammes pourtant spectaculaires semblent n'intéresser personne: les promeneurs rapetissés poursuivent leur chemin. Si l'on n'est guère surpris par les scènes de nuit, avec les trous creusés au milieu de forêts par des figurants à l'allure banale dont on ne sait trop s'ils sont victimes ou bourreaux, c'est parce qu'on les a vues dans des dizaines de films. Plus loin, la vision d'un couple nu allongé sur un matelas douteux au milieu d'un petit jardin mal tenu renvoie à des expériences plus directes de sexe ordinaire: l'homme dort, la femme réfléchit. Parmi les scènes d'intérieur, il en est une qui semble calculée pour devenir emblématique : dans une salle à manger impeccable, sombre et sinistre, une mère et son fils adolescent sont attablés, le regard plongé dans le vide, tandis que le rôti de bœuf refroidit sur la table mise pour quatre, et qu'à l'arrière-plan 
on voit en pleine lumière une cuisine verte aux rideaux orangés, et tout au fond, à travers la fenêtre, une autre maison éclairée de l'intérieur, identique ${ }^{6}$.

C'est la marque de fabrique des productions de Gregory Crewdson qui apparaît ici : des éclairages multiples et complexes (on est loin des lumières naturelles de la «straight photography»), des mises en abyme fréquentes, des profondeurs de champ hyperboliques, sans parler des détails signifiants que Crewdson choisit d'inclure dans ses images, comme par exemple le sang menstruel qui coule du sexe de la femme nue au cheveux gris se tenant devant sa douche, ou l'élan au bord de la rivière, face à l'homme qui l'observe de l'autre côté de la rue enneigée, dans la lumière d'un crépuscule d'hiver. Parfois, l'accumulation des procédés n'empêche pas un certain effet poétique : ainsi ces deux garçons, l'un saisi de dos au retour de l'école, contemplant la femme nue qui se tient dans la porte entrouverte d'une caravane, et l'autre en maillot vert, perdu dans une jungle post-industrielle, le visage levé vers un pont métallique abandonné, au moment précis où tombe sur lui un rayon de lumière à la Spielberg7.

6 Ces images «sans titre» techniquement parfaites, savamment produites et retravaillées numériquement revendiquent une filiation photographique, cinématographique, ou picturale et ce faisant misent beaucoup sur l'effet de charge culturelle maximale qu'elles sont censées produire. Le problème, c'est qu'elles n'y parviennent pas nécessairement, car elles provoquent assez vite une sorte de malaise esthétique, un sentiment d'inadéquation entre les intentions abondamment affichées et la perception que l'on a du résultat. Ce malaise, qu'il convient distinguer de l'intention déstabilisatrice du titre de l'exposition («Uncanny/Unheimlich»), pourrait se décomposer en trois éléments.

7 D'un point de vue global, philosophique si l'on veut, on pourrait dire que s'étale ici la mentalité du fabricateur, de l'artisan qui vient de réaliser l'œuvre. C'est le «banausisme » dont parle Hannah Arendt et qui a pour effet d'entraver la discussion et la formulation d'un jugement : qu'il s'agisse des processus de fabrication, de l'intertexte ou de l'inter-pictorialité volontaires, des causes et des effets, du sens et de l'interprétation, tout est fourni pas l'artiste lui-même, et il ne reste que peu d'espace pour l'appréhension personnelle de l'œuvre, pour le discours critique, pour le jugement esthétique: "la plus grande menace contre l'existence de l'œuvre achevée naît précisément de la mentalité qui l'a produite» disait Hannah Arendt ${ }^{8}$. Dans le cas de Crewdson, on pourrait dire que la menace est largement mise à exécution.

On constate également - c'est la dimension «sociologique» du malaise - que le spectateur est comme submergé par la conjonction d'un ensemble presque irrésistible de forces de légitimation, qu'elles soient universitaires (l'auteur des œuvres enseigne la photographie à Yale), muséales (le Musée Burda l'a jugé digne d'être présenté en regard d'un grand sculpteur récemment disparu), éditoriales (le livre rétrospectif publié par Hatje Cantz, éditeur d'art mondialisé basé en Allemagne, non loin de Stuttgart, accompagne l'exposition), ou commerciales (la galerie Gagosian). Ces légitimations institutionnelles et commerciales sont relayées par une abondante littérature institutionnelle ou journalistique, cette dernière souvent factuelle, assez répétitive et d'une tonalité positive, à quelques exceptions près. Parmi les titres des quelque 200 articles de journaux ou de magazines que l'on peut identifier en recoupant les ressources disponibles ${ }^{9}$, on peut relever ceux-ci, datant tous de l'année 2005, parce qu'ils esquissent une ambiance internationale en parfaite conformité avec les intentions du producteur: "Desperate House Lives ", "Un quotidien de cauchemar " 
[Alptraum Alltag], «Quand la peur se répand » [Quando scatta la paura], « La face noire du rêve américain $»^{10} \ldots$ Le libre marché de la création et des idées rejoint ainsi, en le confortant, le marché de l'art.

9 Mais les images de Crewdson, qu'il s'agisse de la sélection tirée du cycle Beneath the Roses présentée à Baden-Baden ou des trois séries précédentes, posent surtout des questions esthétiques : la plus évidente concerne la validité des filiations revendiquées. On se demande s'il suffit d'égrener les (grands) noms de Walker Evans, de Diane Arbus, de Stephen Shore, de Robert Frank, d'Edward Hopper, d'Alfred Hitchcock, de David Lynch et de quelques autres pour produire à son tour une synthèse aussi canonique dans les intentions qu'improbable dans les faits ${ }^{11}$ ? Pour s'en tenir au strict domaine de la photographie, n'y a-t-il pas quelque hubris à placer ensemble Walker Evans, Robert Frank et Diane Arbus dans la liste des inspirateurs tout en faisant presque exactement le contraire de ce que nous ont laissé ces maîtres? Plus classiquement, on se demande comment situer Crewdson dans la tradition photographique américaine. Appartient-il uniquement à la tradition de la mise en scène photographique (staged photography), aussi ancienne que la photographie elle-même, ou faut-il voir chez lui la mise en œuvre d'un contrat néo-pictorialiste ? Crewdson, à l'instar de Gertrude Käsebier en son temps, cherche-t-il à dire l'indicible avec les moyens limités de la photographie, au prix de quelques manipulations, aussi coûteuses soient-elles sur le plan financier? Mais une œuvre peut-elle encore exister lestée d'un tel surpoids de citations, d'influences revendiquées aussi contradictoires qu'incompatibles? Et que faire des nombreuses références à la culture populaire hollywoodienne et télévisuelle, qui se matérialisent notamment par les apparitions d'acteurs connus comme Julianne Moore, William Macy ou Susan Sarandon dans des tableaux figés et glaçants ${ }^{12}$ ?

On note enfin le problème posé par l'enveloppe psychanalytique reprise dans le titre choisi par le musée Burda : L'inquiétante étrangeté du réel/Uncanny Realities/Unheimliche Wirklichkeiten. Celle-ci suffit-elle à conférer une cohérence à l'ensemble des séries produites par Crewdson? On nous explique que "les photographies de Crewdson tournent autour d'un unique thème général : le retour du refoulé, de l'étrange et de l'inexplicable dans un monde prétendument intact et protégé $»^{13}$. Crewdson illustrerait donc les analyses de Freud dans Das Unheimliche, article classique publié en 1919, où il tente de cerner la nature profonde du refoulement. Il faut dire Crewdson ne lésine guère sur les moyens pour nous rappeler que nous sommes ici sur le terrain de la psychanalyse freudienne: dans bien des images, on retrouve un attirail psychanalytique au charme désuet ou grand guignolesque, selon les situations oiseaux, papillons, animaux sauvages, végétaux incontrôlables, taches, sang, fenêtres, miroirs, flammes, eau ${ }^{14}$.

11 Crewdson, on l'a dit, bénéficie d'une réception critique qui paraît globalement positive, comme s'il existait un consensus presque automatique ou obligé autour de cette œuvre définie et délimitée avec tant de soin dès le stade de la production. La plupart du temps, on reconnaît et on salue d'une manière un peu sentencieuse l'illustrateur du côté sombre du "rêve américain", le révélateur de tous les refoulés sociaux et culturels d'une société malade. Le texte du dépliant proposé par le Musée Burda constitue à cet égard une synthèse caractéristique et éclairante du point de vue moyen sur l'œuvre de Crewdson :

Pour la réalisation de ses œuvres, Crewdson sillonne l'État du Massachusetts à la recherche de lieux d'atmosphère: il y installe ses décors, qui sont de véritables plateaux de tournage. Les habitants de ces petites villes lugubres sont souvent 
invités à incarner eux-mêmes les personnages de la mise en scène. Les photographies de Crewdson évoquent ainsi des images de film et, de fait, l'artiste est très proche de l'art cinématographique ${ }^{15}$.

l'emploi de l'adjectif "lugubre" fait ici écho à certains détails insistants des photographies elles-mêmes, comme ces feux obstinément bloqués sur l'orange dans plusieurs images de carrefours déserts au petit matin. Un peu plus loin, pour ceux qui ne disposeraient pas des outils nécessaires, on trouve cette orientation biopsychologique permettant de conférer à l'œuvre dans son ensemble le sens qui lui revient :

Les images de Crewdson sont un travail très concerté autour d'émotions et d'angoisses dont son œuvre offre, sous des scénarios variés, les images récurrentes : isolement, absence, honte, désir, frustration - des sentiments qui touchent le spectateur au plus profond de lui-même. Que Crewdson travaille ainsi au plus près de la psyché tient peut-être aussi au fait que ce fils de psychanalyste a été familiarisé de bonne heure avec la complexité troublante de l'âme humaine ${ }^{16}$.

Il y a des exceptions à ce consensus, des commentaires plus circonspects. Dès 2000, dans un article consacré à l'opération Twilight, un critique se déclarait « déçu par tant d'eau tiède $\aleph^{17}$. Dans Time en 2007, Richard Lacayo s'en prend à la dérive hollywoodienne de Crewdson, c'est-à-dire au recours excessif aux acteurs de cinéma qui viennent peupler certaines images, mais aussi plus généralement à la confusion des genres cinématographique et photographique :

Les images de Crewdson cessent de fonctionner au moment précis où vous vous demandez quand aura lieu la fête de la fin du tournage. Et nous y sommes. La mise en scène photographique, lorsqu'elle est faible, ne résiste pas à la tentation d'imiter les mondes fabriqués que nous voyons tous les jours au cinéma et dans les publicités ${ }^{18}$.

Arrêtons-nous un instant sur la contradiction entre les ambitions artistiques de Crewdson et le résultat finalement banal et convenu, cette " eau tiède " dont parle le critique clairvoyant: cette tension produit un effet de mensonge esthétique, une impression de tromperie sur la marchandise, de facilité, bref un ensemble de caractéristiques qui sont celles du kitsch, mot et concept apparus à Munich dans les années 1880 : il s'agissait alors de désigner les petits tableaux exécutés à la va-vite pour les vendre rapidement à un prix modique - c'était le sens du verbe «verkitschen $»^{19}$. Bientôt, le mot kitsch s'installe dans la langue allemande avec le sens de "pacotille de mauvais goût pour les masses $»^{20}$, tout ce qui modifie les dimensions, les proportions, les couleurs au bénéfice des effets faciles et de la sentimentalité. À l'occasion de son adoption en anglais, probablement sous l'influence des philosophes de l'école de Francfort réfugiés aux États-Unis, le mot continue de désigner le mauvais goût et l'absence de valeur artistique, mais il renvoie désormais aussi aux effets aliénants de la culture de masse qui y est assimilée (films hollywoodiens, comics et musique populaire), comme en attestent les essais célèbres d'Irving Howe ou de Dwight Macdonald ${ }^{21}$. Le mot kitsch est aujourd'hui assez courant en français, dans une acception large et légèrement modifie, puisqu'elle intègre un point de vue prudemment relativiste : à l'entrée correspondante, le Petit Robert (édition 2000) parle « d'un style ou d'une attitude esthétique caractérisés par un ensemble hétéroclite d'éléments démodés ou populaires considérés de mauvais goût par la culture établie et produits par l'économie industrielle ». 

points au moins. "L'ensemble hétéroclite d'éléments démodés ou populaires » est constitué par le mélange référentiel qui caractérise la quasi-totalité des images de Crewdson. Au catalogue thématique familier - réalités suburbaines, intérieurs glauques, nus réalistes, érotisme ordinaire, violence contenue - dans lequel ont abondamment puisé la littérature, le cinéma et plus récemment la bande dessinée américaine, s'ajoute un habillage idéologique sans surprise ("le rêve américain ", et son côté «sombre" ou son inversion en cauchemar). Seuls les fantasmes érotiques adolescents mis en scène par Crewdson échappent peut-être à ce catalogue «d'éléments démodés ou populaires » - mais ils renvoient à des entreprises autrement novatrices, risquées et troublantes, comme les photographies et les films de Larry Clark (né en 1943) ou la peinture d'Eric Fischl (né en 1948) ${ }^{22}$. Quant au critère de la "production par l'économie industrielle », il est en partie pertinent, puisque Crewdson "produit» chaque image comme s'il s'agissait d'un film, sans parler de l'insertion délibérée dans le marché de l'art, déjà évoquée. d'affirmer que la "culture établie » juge de "mauvais goût» le travail de Crewdson. C'est même tout le contraire, en apparence du moins, puisque les institutions, les journaux, le marché de l'art portent sur cette œuvre une appréciation positive, tandis qu'aucun texte critique, sauf erreur, n'évoque le kitsch à son propos. Il est pourtant difficile de nier que la plupart des images produites par Crewdson partagent une caractéristique fondamentale du kitsch, à savoir celle d'un recyclage permanent et systématique d'éléments visuels ou discursifs aisément reconnaissables: comme le notait l'essayiste autrichien Hermann Broch, pour qu'il y ait du kitsch, il faut aussi qu'il $\mathrm{y}$ ait des «Kitschmenschen» qui en ont l'usage ${ }^{23}$. C'est l'accessibilité immédiate de ces images et l'impression de familiarité qui émane d'elles qui constituent leur fragilité principale. Quelle part de surprise, de mystère, de doute, de profondeur reste-t-il ? Pour le philosophe Vilem Flusser, le kitsch, phénomène passager, doit être compris comme un phénomène d'encombrement dans le processus de "recyclage du rebut " (Abfall Recycling) caractéristique selon lui du passage de la société industrielle à la société de l'information: le kitsch "exprime le refus d'affronter directement l'absurdité de l'existence, et la volonté de mourir confortablement» (gemütlich sterben) ${ }^{24}$.

17 Pour finir, il est intéressant d'évoquer brièvement deux textes d'écrivains, pièces majeures du puzzle de la légitimation sociale, esthétique et commerciale du travail de Crewdson. En 2002, Rick Moody, romancier reconnu qui aime et connait la photographie, écrit l'introduction du livre intitulé Twilight, tandis que Russell Banks, autre romancier célèbre, fait de même pour Beneath the Roses en 2008.

Pour Rick Moody - on ne se refait pas - Crewdson est avant tout un matériau. Moody brosse un tableau des origines de l'artiste, de sa famille aisée vivant à Park Slope, quartier résidentiel de Brooklyn. Il imagine les sorties dominicales de l'enfant Crewdson en compagnie de son père psychanalyste, et tout particulièrement une visite de la rétrospective de Diane Arbus ou Moma en 1972, puis il chemine dans le paysage mental d'un adolescent des années 70 : les premières expériences amoureuses sur le divan du père absent, Patti Smith, la télévision et les films d'épouvante tard dans la nuit. Il évoque le bref passage de Gregory Crewdson dans le rock punk/pop, à l'époque où il est guitariste et compositeur d'un groupe ayant joui d'une notoriété locale à la fin 
des années 1970, the Speedies ${ }^{25}$, puis le séjour à Purchase College. C'est apparemment là que s'opère la transformation du jeune rocker en artiste et "artificier démoniaque ». Pour Moody, l'expérience de Crewdson est structurée par la contradiction, entre les promesses idéales des suburbs et les désordres réels de la vie psychique et sociale, entre l'attirance pour le bucolique et les dangers de la nature sauvage, entre la vie et l'ordre.

Russell Banks, pour sa part, s'engage dans un essai d'une tonalité plus démonstrative, à propos du cycle suivant, Beneath the Roses. Cherchant àdéfinir la nature de l'art de Crewdson, Banks, qui procède par approfondissements successifs, nous diten substance ceci : on dirait du cinéma, mais ce n'est pas du cinéma. Les images de Crewdson font plutôt penser, poursuit-il, à la fiction, car les photographies sont «incomplètes » et inscrites dans une série. "Cartographe du quotidien", Crewdson, est "l'explorateur des petits matins, l'arpenteur des ruines de notre Nouvelle Jérusalem, de notre Cité sur la Colline, de notre Nouveau Monde » et, inévitablement, de «tout ce qui a transformé le Rêve Américain en cauchemar ${ }^{26}$. Pour Russell Banks, Crewdson s'exprime par séries et n'est pas vraiment un photographe, ni même un metteur en scène ou un producteur, mais plutôt un "homme de spectacle unique en son genre, sans pareil » : producteur, auteur et acteur d'un spectacle total, d'une sorte "d'opéra " qui serait représenté parfois « dans de grandes galeries ou des musées en grande pompe avec un orchestre complet et un chœur, parfois d'une manière plus modeste comme ici, dans un livre ${ }^{27}$ d'où les aspects mélodramatiques, tous les éléments d'accompagnement, un spectacle qui ne cache rien, de la première idée au résultat final, en passant par toutes les étapes de la fabrication.

On peut être d'accord avec les deux: comme le dit Moody, il y a dans le travail de Crewdson tous les éléments d'une recréation d'une certaine atmosphère suburbaine des années 1970. Ses productions sont autant de mises en scène du retour dans le familier d'un refoulé supposé inquiétant et qui fait (un peu) peur: "Quando scatta la paura" disait le magazine italien... Et l'on peut également accepter la proposition de Russell Banks qui voit ici une œuvre mélangée proposant un spectacle total où tous les détails comptent, sans hiérarchie, la coulisse pas moins que la scène. Mais on peut penser aussi que l'opération de saturation tous azimuts - qui vend à la fois de l'image, de la couleur, du sens, un peu de sexe amateur, du refoulé et de l'inconscient, de la valeur artistique proclamée et une valeur commerciale avérée, court le risque de l'épuisement. Et en effet, dans la dernière série de Crewdson, publiée sous le titre Sanctuaire, on trouve

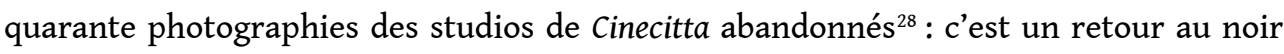
et blanc, mais l'effet est gris. L'ouvrage correspondant, d'un format équivalent aux précédents, semble tout à coup encombrant. De la saturation et du trop-plein, on est tombé dans le vide, comme s'il n'y avait plus rien à photographier que ces décors devenus inutiles, absurdes. 


\section{NOTES}

1. La galerie Gagosian donne une liste complète sur son site http://www.gagosian.com. Voir aussi http://www.artnet.fr.

2. Hans-Michael KOETZLE, Photographes A-Z, Cologne, Taschen, 2011, 83.

3. Stephan BERG (dir.), Gregory Crewdson 1985-2005, Ostfildern, Hatje Cantz, 2006. [Textes en anglais et en allemand]. Cet ouvrage comprend également une liste des expositions et une bibliographie.

4. Gregory CREWDSON, Rick MOODY, Darcey STEINKE, Joyce Carol OATES et Bradford MORROW, Hover : Photographs, San Francisco, Artspace Books, 1998 ; Gregory CREWDSON et Rick MOODY, Twilight: Photographs by Gregory Crewdson, New York, Harry N. Abrams 2002 ; Gregory CREWDSON et Russell BANKS, Beneath the Roses, New York, Harry N. Abrams, 2008.

5. Outre les ouvrages mentionnés ci-dessus, de nombreux sites internet donnent un aperçu du travail photographique de Crewdson. Nous nous permettons d'y renvoyer le lecteur.

6. On retrouve cette photographie dans la brochure qui accompagnait les représentations de la pièce Conversations avec ma mère de Santiago Carlos Ovès, dans la mise en scène de Didier Bezace au cours de la saison 2010-2011 du Théâtre National de Strasbourg, p. 7, en regard d'une citation de Stig Dagerman tirée de L'Enfant brûlé, roman publié en France en 1981 : «... Ils savent qu'ils sont mère et fils, il le savent les yeux ouverts...».

7. Toutes les images décrites ou commentées ici figurent dans Gregory CREWDSON et Russell BANKS, Beneath the Roses, op. cit. Cf. planches 1, 10, 11, 21, 22 (carrefours) ; 12 (supérette) ; 7, 17, 18, 20, 40 (intérieurs avec salles de bains) ; 4 et 15 (voies de chemin de fer désaffectées) ; 5, 6 (forêts) ; 16, 30, 35 (sexe ordinaire) ; 19 (mère et fils) ; 18 (sang menstruel) ; 25 (élan) ; 13, 29 (petits garçons).

8. Hannah ARENDT, «La crise de la culture », dans La crise de la culture, Paris, Gallimard, 1972, 275-76.

9. Outre les ouvrages mentionnés ci-dessus, on peut consulter la sélection proposée sur le site de la galerie Gagosian, ou sur http://rogallery.com/Crewdson/Crewdson-bio.html.

10. Tony ALLEN-MILLS, «Desperate House Lives », The Sunday Times Magazine, 8 avril 2005, 4049 ; «Alptraum Alltag », Photographie-Das Magazin für digitale und analoge Photographie, octobre 2005 ; Germano CELANT, «Quando scatta la paura», L'espresso, 20 octobre, 2005 ; Michel GUERRIN, « La face noire du rêve américain », Le Monde, 9 décembre 2005.

11. Voir par exemple Gregory CREWDSON, «In a Lonely Place », Aperture n 190, Printemps 2008, 79-86.

12. Dans la série Dream House essentiellement.

13. Cet extrait d'un texte de Stephan Berg figure dans le dictionnaire de Hans-Michael Koetzle, op. cit. Voir aussi Stephan BERG, "The Dark Side of the American Dream », dans Stephan BERG, Gregory Crewdson 1985-2005, op. cit. 11.

14. Richard Lacayo fait un constat similaire dans son article sur la mise en scène photographique, "If you build it, they will come", Time, 5 mars 2007, vol. 169, numéro 10, p. 68-72 15. Dépliant Musée Burda, sans mention de nom d'auteur.

16. Ibid.

17. "It's disappointlingly weak tea ». David FRANKEL, "Gregory Crewdson", Artforum International 38.10, 22 juin 2000.

18. "Crewdson's pictures stop working the minute you find yourself wondering about the wrap party. But there it is. At its weakest, staged photography succumbs to the temptation to imitate the staged worlds we see every day in movies and advertising ». Cf. Richard Lacayo, op. cit. 
19. Cf. Harry PROSS, « Kitsch oder nicht Kitsch?», dans Harry PROSS (dir.), Kitsch : Soziale und Politische Aspekte einer Geschmacksfrage, Munich, List Verlag, 1985, 19.

20. «Geschmackloser Massenschund ». La formule est due à l'historien d'art et directeur de Musée Gustav Pazaurek (1865-1935) dans son livre Guter Und Schlechter Geschmack Im Kunstgewerbe, publié à Berlin et Stuttgart en 1912.

21. Dwight MACDONALD, “A Theory of Mass Culture" (1944) et Irving HOWE, "Notes on Mass Culture" (1948), textes reproduits dans Bernard ROSENBERG et Daniel W. WHITE (dir.), Mass Culture: The popular arts in America, New York, Macmillan, 1957.

22. Sauf erreur, Eric Fishl fut le premier peintre figuratif américain à s'aventurer sur ce terrain dans ses toiles de grandes dimensions, et ce dès les années 1980.

23. Cité par Harry PROSS, op. cit. 23.

24. Vilem FLUSSER, "Gespräch, Gerede, Kitsch: Zum Problem des unvollkommenen Informationskonsums", dans Harry PROSS, op. cit. 61-62.

25. On trouve sur Youtube une intéressante vidéo datée de 1979, dans laquelle les Speedies chantent «Let me take you photo ». Le chanteur, qui pourrait être Gregory Crewdson, arbore un accent britannique marqué.

26. "Cartographer of the quotidian" [...] "He's digging through the ruins of $5: 00$ A.M. [...] ; he's mapping and measuring the tumbled down cellar walls of our New Jerusalem, our City on a Hill, our New World, [...] everything that turned the American Dream into the American nightmare". Cf. Gregory CREWDSON et Russell BANKS, op. cit. 7-8.

27. "Crewdson is sui generis, inimitable..." [...] “...it is an opera, one performed sometimes in a large gallery or museum in grand style with full orchestra and chorus, as it were, or sometimes more modestly like this, in a book". Cf. Gregory CREWDSON and Russell BANKS, op. cit. 9.

28. Gregory CREWSDON, Sanctuary, New York, Abrams, 2010.

INDEX

Thèmes : Trans'Arts

\section{AUTEUR}

\section{BERNARD GENTON}

Université de Strasbourg 